\title{
Correction to: Daratumumab: A Review in Combination Therapy for Transplant-Eligible Newly Diagnosed Multiple Myeloma
}

\author{
Yvette N. Lamb ${ }^{1}$
}

Published online: 17 December 2020

C) Springer Nature Switzerland AG 2020

\section{Correction to: Drugs (2020) 80:1455-1464 \\ https://doi.org/10.1007/s40265-020-01385-x}

\section{Correction in reviewer name:}

Page 1455: The listing of the reviewer names and affiliations, which previously read:

N. Callander, University of Wisconsin Carbone Cancer Center, Madison, WI, USA; C. Cerchione, Hematology Unit, Istituto Scientifico Romagnolo per lo Studio e la Cura dei Tumori (IRST) IRCCS, Meldola, Italy; S. Knop, Medizinische Klinik und Poliklinik II, Universitätsklinikum Würzburg, Würzburg, Germany; S. J. Kumar, Hematology and Internal Medicine, Mayo Clinic, Rochester, MN, USA. should read:

N. Callander, University of Wisconsin Carbone Cancer Center, Madison, WI, USA; C. Cerchione, Hematology Unit, Istituto Scientifico Romagnolo per lo Studio e la Cura dei Tumori (IRST) IRCCS, Meldola, Italy; S. Knop, Medizinische Klinik und Poliklinik II, Universitätsklinikum Würzburg, Würzburg, Germany; S. K. Kumar, Hematology and Internal Medicine, Mayo Clinic, Rochester, MN, USA.

The original article can be found online at https://doi.org/10.1007/ s40265-020-01385-x.

Yvette N. Lamb

demail@springer.com

1 Springer Nature, Mairangi Bay, Private Bag 65901, Auckland 0754, New Zealand 